\title{
O USO DA ATENÇÃO INTERMEDIÁRIA COMO PORTA DE ENTRADA PREFERENCIAL AO SUS: A PERCEPÇÃO DOS USUÁRIOS CLASSIFICADOS COMO NÃO URGENTES NA UPA 24 HORAS DR. VALDIR DE CAMARGO, BRAGANÇA PAULISTA, SP
}

THE USE OF BASIC CARE AS PREFERENTIAL PORT OF ENTRY TO SUS: THE PERCEPTION OF THE USERS CLASSIFIED AS NOT URGENT AT UPA 24H HOURS DR. VALDIR DE CAMARGO, BRAGANCA PAULISTA, SP

\section{PAES, Karina da Silva Mota ${ }^{1}$ NASCIMENTO, Joni Carlos do ${ }^{2}$ NEGRINI, Lisamara Dias de Oliveira $^{3}$ \\ ${ }^{1}$ Universidade São Francisco; ${ }^{2}$ Universidade São Francisco; ${ }^{3}$ Universidade São Francisco kasipaes@hotmail.com}

RESUMO. O Sistema Único de Saúde - SUS tem a Atenção Básica como a porta de entrada preferencial. No entanto, serviços de urgência e emergência são utilizados, com frequência, como porta de entrada ao sistema por pacientes considerados não urgentes. Objetivos: identificar as razões que induzem os usuários a optarem pelos serviços de urgência/emergência como porta de entrada preferencial, identificar o grau de importância e resolutividade que os usuários atribuem ao SUS, avaliar o nível de conhecimento da população sobre o que é o SUS e o grau de satisfação dos mesmos. Trata-se de uma pesquisa descritiva, exploratória, qualitativa, cuja coleta de dados ocorreu na UPA 24hs Dr. Valdir de Camargo, com os usuários classificados como não urgentes, com idade a partir de 18 anos. Identificou-se que os entrevistados, em sua maioria, não possuíam conhecimento sobre o que é o SUS, optavam primeiramente pelo atendimento na UPA devido a UBS/ESF possuir horário de atendimento limitado e somente agendado e considerarem que a UPA apresenta maior resolutividade. Os níveis de atenção secundário e terciário foram considerados melhores em relação à $\mathrm{AB}$ quanto à importância e capacidade de resolução. 52,67\% dos entrevistados julgaram bons os serviços oferecidos pelo SUS e 84,67\% os recomendaria. Acredita-se que este estudo poderá contribuir na avaliação do desempenho atual do sistema de saúde, dos profissionais de saúde e gestores, mobilizando iniciativas que levam ao remodelamento da atenção à saúde, possibilitando o fortalecimento da Atenção Básica e melhor conhecimento da população sobre o que o Sistema Único de Saúde representa.

Palavras-chave: Sistema Único de Saúde, atenção básica, urgência, emergência.

ABSTRACT. The Unified Health System - SUS has basic care as a preferential point of entry. By the way, urgency and emergency services are frequently used as a point of entry by patients that are not considered urgent. Objectives: indentify the reasons that empower the users to choose emergency services as preferential point of entry, identify the degree of importance and problem-solving that the users attach to SUS, evaluate the level of knowledge of the population about what is SUS and the degree of satisfaction of both. It's all about a descriptive, exploratory and qualitative research, which data collection occurred at UPA 24hs Dr. Valdir de Camargo with not urgent classified users with age as of 18 years. Were indentified that the interviewed hadn't knowledge about what is SUS. They chose primarily for service at UPA because UPA/ESF has limited opening hours and only scheduled and consider that UPA has more problem-solving. The second and third level of were considered 
better than $\mathrm{AB}$ about importance an capacity of resolution. 52,67\% of the interviewed judged well the services offered by SUS, and $84,67 \%$ would recommend it. It is believed that this study could contribute on the evaluate of the actual performance of health system, of health profissionals and managers, mobilizing initiatives that leads at remodeling of health care, possibiliting the strengthening of the basic care and better knowledge of the population about what is Unified Health System represents.

Keywords: Unified Health System, basic care, urgency, emergency.

\section{INTRODUÇÃO}

O Sistema Único de Saúde - SUS representa um dos maiores sistemas públicos de saúde existentes, tem seus preceitos datados de 1988, garante o acesso universal, integral e gratuito para a população brasileira. O cuidado em saúde no âmbito do SUS foi estruturado em níveis de atenção, ou seja, assistência básica, média e alta complexidade, de maneira a programar e planejar as ações e serviços do sistema de saúde, e assim potencializar os recursos disponíveis (BRASIL, 2011; BRASIL, 2009; NARVAI, 2005).

A Atenção Básica - AB compõe o primeiro nível de atenção, sendo configurada como porta de entrada ao sistema de saúde, estabelecendo acesso preferencial à população e capaz de resolver mais de $80 \%$ dos problemas de saúde (PAIM, 2015). Gomide, Pinto e Figueiredo (2012) ainda complementam que a $\mathrm{AB}$ assume a organização do sistema em uma rede articulada e unificada. No entanto, verifica-se que serviços de urgência e emergência, ordenados em nível intermediário de assistência, estão atendendo pacientes com problemas sensíveis de serem solucionados pela Atenção Básica. Isto é reafirmado por Freire et al. (2015) ao argumentar que serviços de caráter emergencial são utilizados em alto grau como porta de entrada ao sistema de saúde pelos pacientes considerados não urgentes.

Em serviços de urgência e emergência, o acolhimento aos pacientes é delineado pela classificação de risco, sendo correligionária a um protocolo direcionador e definida como um processo de identificação dos casos que necessitam de assistência imediata consoante ao potencial de risco e nível de sofrimento. Dentre os protocolos de classificação de risco, o Protocolo de Manchester é o utilizado pela maioria dos estados brasileiros e classifica os pacientes em cinco níveis de prioridade de atendimento: nível 1 - emergente, nível 2 - muito urgente, nível 3 - urgente, nível 4 - pouco urgente e nível 5 - não urgente (SOUZA; ARAÚJO; CHIANCA, 2015). A classificação de pacientes foi instituída devido ao aumento dos fluxos dos usuários nos serviços de prontidão, uma vez que acarretou na necessidade de reestruturar a metodologia de trabalho, de forma a priorizar a situação clínica para o atendimento (GOMIDE; PINTO; FIGUEIREDO, 2012).

O acesso equivocado aos serviços de saúde do SUS tende a ter relação com o conhecimento insuficiente sobre o sistema pelos usuários, bem como, a não flexibilidade dos serviços à demanda espontânea e as próprias expectativas do usuário quanto à política de ação do SUS. Tal dinâmica de acesso minimiza a eficácia e eficiência de serviços especializados na resolução dos problemas de saúde, sendo necessário à Atenção Básica prover melhor organização do fluxo de usuários no sistema de saúde.

O interesse em realizar esta pesquisa surgiu da observação do perfil dos usuários que procuravam atendimento nos serviços assistenciais de urgência e emergência, durante os estágios curriculares do curso de graduação. O presente trabalho se propõe a identificar os fatores que levam os usuários do Sistema Único de Saúde a optarem pelos serviços de urgência e emergência como porta de entrada prioritária para a resolução de agravos de saúde não urgentes e ainda contribuir para o entendimento das Diretrizes do Sistema Único de Saúde, assim como sua aplicação prática. 


\section{METODOLOGIA}

Este estudo caracterizou-se como uma pesquisa descritiva, exploratória, de abordagem qualitativa. Concentrou na Unidade de Pronto Atendimento 24 horas Dr. Valdir de Camargo, localizada na Rua Sebastião Cícero Franco, no município de Bragança Paulista, estado de São Paulo.

A população de estudo representou os usuários que buscaram atendimento e foram classificados como casos não urgentes, sinalizados pela cor azul, conforme protocolo de classificação de risco. A amostra representou o equivalente a $1 \%$ do total geral de atendimentos mensais realizados pelo serviço. Foram incluídos na pesquisa usuários do sexo masculino e feminino, com faixa etária igual ou superior a 18 anos, residentes ou não no município de Bragança Paulista, sendo excluídos os que não atenderam a faixa etária, assim como os acompanhantes dos usuários em questão.

Foi utilizada para pesquisa a base de dados SciELO, Biblioteca Virtual em Saúde, Legislações Federais, livros em formato impresso e digital e busca manual de artigos, limitando-se às publicações entre 1988 e 2017 no idioma português.

O desenvolvimento da entrevista foi previamente esclarecido e autorizado pela assinatura de Termo de Consentimento Livre e Esclarecido (Apêndice C), submetido e aprovado pelo Comitê de Ética em Pesquisa - CEP. Os usuários da unidade foram submetidos a uma entrevista com formulário estruturado (Apêndice B) composto por questões objetivas e dissertativas, onde se buscou identificar os motivos que levaram estes usuários a optarem pelos serviços de urgência e emergência como porta de entrada preferencial, em detrimento da Atenção Básica. A coleta de dados foi realizada durante o mês de Julho de 2017 e a análise dos dados foi realizada através de tabelas e gráficos.

\section{RESULTADOS E DISCUSSÃO}

TABELA 1 - Motivos pela opção de atendimento na UPA. Bragança Paulista, 2017. $(\mathrm{N}=150)$.

\begin{tabular}{lcc}
\hline Domínio & Frequência & $\%$ \\
\hline UBS/ESF somente atendimento agendado & 69 & 46,00 \\
UPA possui maior resolutividade & 27 & 18,00 \\
UBS/ESF horário limitado de atendimento & 25 & 16,67 \\
UPA possui atendimento rápido & 10 & 6,67 \\
UPA serviço mais próximo a residência/trabalho & 07 & 4,67 \\
UPA possui qualidade de atendimento superior & 07 & 4,67 \\
UPA indicada por amigos/familiares & 02 & 1,33 \\
UBS/ESF não recordada sua existência & 02 & 1,33 \\
UPA referência de atendimento para não residentes & & 0,67 \\
do município & 01 & $100 \%$ \\
\hline Total & 150 &
\end{tabular}

Fonte: Próprio autor.

Pelos dados apresentados na tabela 1, nota-se que os motivos prevalentes para a procura à UPA foram: atendimento na UBS/ESF de caráter apenas agendado $(46,00 \%)$, a UPA apresentar maior resolutividade $(18,00 \%)$ e horário de funcionamento da UBS/ESF $(16,67 \%)$. Os menos referidos foram: indicação de amigos e familiares para uso da UPA $(1,33 \%)$, não se recordarem da existência da UBS/ESF $(1,33 \%)$ e em virtude da UPA ser referência de atendimento para não residentes do município de Bragança Paulista $(0,67 \%)$.

Gomide, Pinto e Figueiredo (2012) afirmam que a organização dos serviços de saúde é pautada no modelo tecno-assistencial análogo a uma pirâmide, onde a base consiste na 
atenção básica que atua como porta de entrada, o centro é representado por ambulatórios de especialidades e Unidades de Pronto Atendimento que apresentam densidade tecnológica maior, e no ápice encontram-se os hospitais. Para esses autores esta hierarquização na rede de atenção à saúde garante o acesso pela porta de entrada e integralidade dos recursos disponíveis, porém o usuário não se limita a norma estabelecida sobre qual é a sua porta de entrada no sistema, optando pelo serviço que mais the convém e baseado em suas próprias experiências.

De acordo com Oliveira e Scochi (2002), a Unidade de Pronto Atendimento como porta de entrada é uma realidade nos serviços de saúde. Marques e Lima (2007) apontam que a procura pela UPA em situações não urgentes está atrelada ao fato de que estas unidades apresentam um conjunto de recursos, como consultas, medicamentos, procedimentos de enfermagem, exames laboratoriais e radiológicos. O estudo de Pires et al. (2013) menciona que os motivos pelos quais os usuários preferem o atendimento da UPA em detrimento da UBS, relacionam, proximidade da residência, serviço na UPA é melhor do que o serviço próximo a residência, indicação/solicitação de outros serviços de saúde, por decisão própria de família e terceiros e atendimento mais rápido. Dentre os motivos acima, a proximidade da residência e atendimento mais rápido e melhor, condizem com a presente pesquisa.

$\mathrm{O}$ agendamento de consultas na Atenção Básica também foi um dos fatores evidenciados na pesquisa de Gomide, Pinto e Figueiredo (2012) para procura do pronto atendimento como porta de entrada para o acesso aos serviços do sistema de saúde. $\mathrm{O}$ atendimento efetivo e resolutivo pelas UPAs também foi identificado por Freire et al. (2015) como um dos motivos mais prevalentes, relacionando ainda, localização mais próxima da residência, horário de atendimento acessível, usuários se considerarem como situação de urgência e emergência, exame realizado com agilidade, entre outros.

Rocha (2005) afirma que os serviços de urgência representam, para o usuário, unidades para tratamento de doenças, já os Centros de Saúde são unidades de controle das mesmas. Ainda relata que, a procura às Unidades de Pronto de Atendimento é consequência à maior densidade tecnológica juntamente a rapidez e agilidade no atendimento, ou seja, tais unidades possibilitam atendimento médico no mesmo dia e conferem maior resolutividade aos problemas de saúde ao realizarem exames laboratoriais, de imagem e outros. O mesmo autor também identificou os motivos de não procura pelas UBS, dentre eles, atendimento deficiente, falta de confiança no serviço, demora no atendimento e ter de levantar cedo para conseguir passar pelo acolhimento, o que por sua vez, exprime demanda maior que a oferta e carência de médicos.

Para Marques e Lima (2007) a procura por serviços de saúde é influenciada pela gravidade ou a urgência do problema, tecnologia disponível, rapidez no atendimento, nível de resolubilidade, acolhimento e pelas condições de acesso. $\mathrm{O}$ modelo piramidal de atenção à saúde, segundo Monteiro, Silva e Cabral (2008), ainda que tenha levado a transformações importantes na estrutura e processo de trabalho nos serviços de saúde, não alcançou um resultado que contente as necessidades da população. Este modelo, de acordo com Camerro et al. (2015), demanda melhorias na Atenção Básica, pois será em vão orientar os usuários a procurarem o serviço primário se este não funcionar conforme suas necessidades, levando os mesmos às Unidades de Pronto Atendimento, mesmo não caracterizando o perfil de atendimento destas unidades. Rocha (2005) complementa que a Rede Básica não vem cumprindo com o seu dever.

Verifica-se pelo gráfico 1, que o hospital e a UPA foram consideradas as instituições de saúde com maior capacidade de resolução na opinião dos entrevistados, com 59,33\% e $50,67 \%$ respectivamente, enquanto a UBS $10,00 \%$. Observa-se ainda, o predomínio da UBS para a média $(60,00 \%)$ e baixa $(30,00 \%)$ capacidade. 


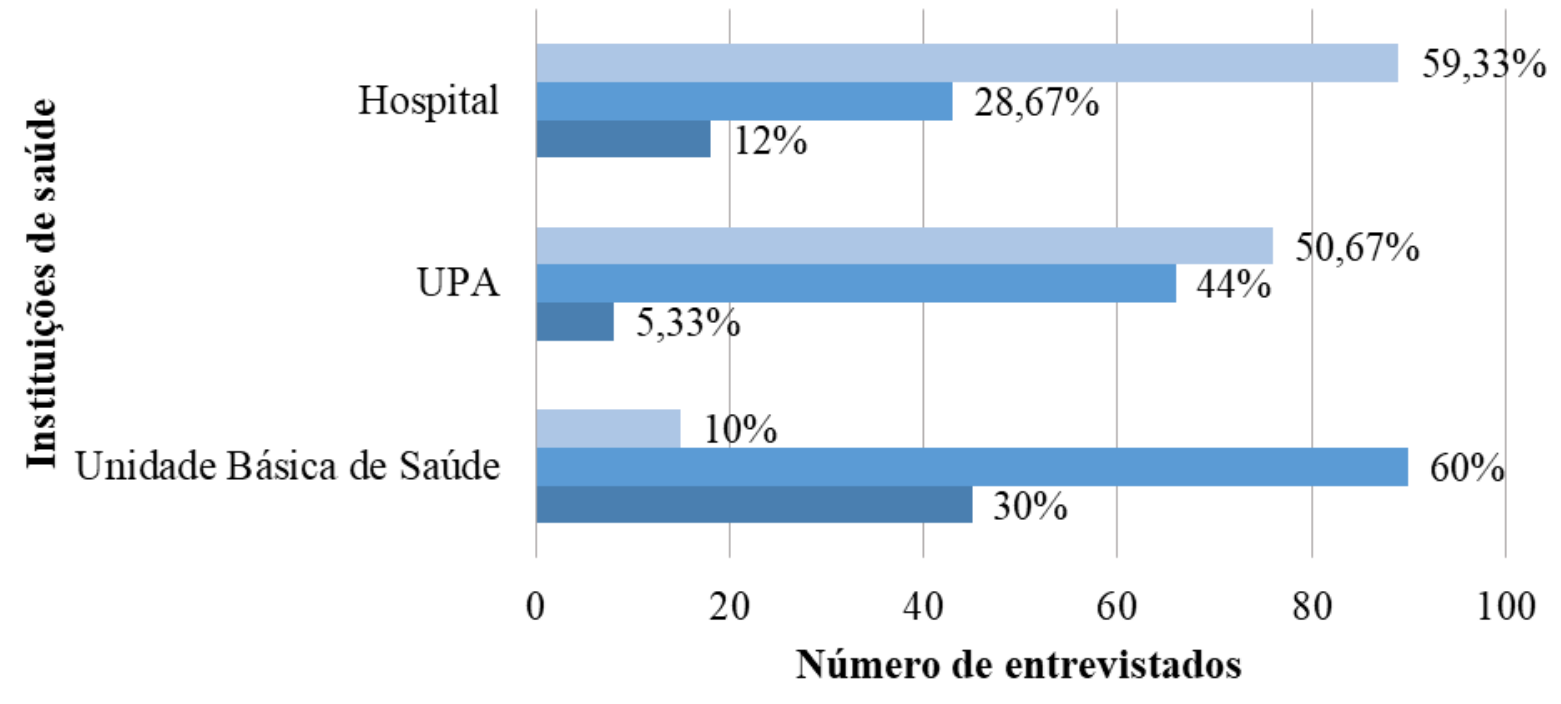

$\square$ Alta Média $\square$ Baixa

Gráfico 1 - Distribuição da capacidade de resolução atribuída às instituições de saúde. Bragança Paulista, 2017. (N=150) (Fonte: Próprio autor).

Segundo Gomide, Pinto e Figueiredo (2012) a UBS, quando funciona adequadamente, consegue resolver a maioria dos problemas de saúde de uma população, contudo, conforme observado no gráfico acima, a maioria dos entrevistados atribui à UBS uma capacidade de resolução mediana, já uma parcela significativamente baixa refere alta capacidade. Apesar de a UBS e ESF serem unidades de saúde diferentes, ambas enquadram-se na Atenção Básica, portanto, é válido fazer menção ao estudo de Chagas e Vasconcellos (2013), onde constatouse que a população não considera a ESF apta a resolver seus problemas de saúde e identifica como fragilidades o acolhimento, recursos humanos desqualificados e desorganização do processo de trabalho, tanto ao nível assistencial como gerencial.

O hospital, nesta pesquisa, é considerado pela maior parte dos entrevistados como detentor de uma alta capacidade de resolução, porém neste contexto, é válido mencionar Baristela, Guerreiro e Rossetto (2008) ao afirmarem que entre a população existe uma crença de que em hospitais o atendimento é melhor, e tal crença, por sua vez, é orientada ao modelo assistencial biomédico, onde os hospitais representam o centro do sistema de saúde e a rede básica, no entanto, é vista como inábil.

Pelo gráfico 2, nota-se que a maioria dos entrevistados avaliou as instituições de saúde, hospital $(70,00 \%)$, UPA $(64,67 \%)$ e UBS $(46,67 \%)$, como sendo muito importantes. Os percentuais de importância foram muito semelhantes entre hospital $(28,67 \%)$ e UPA $(32,67 \%)$ e superiores para UBS $(46,00 \%)$.

A hierarquização é um dos princípios organizativos do Sistema Único de Saúde e, levando em conta Paim (2015), tal princípio não anuncia que um serviço seja mais ou menos importante que o outro, apenas objetiva utilizar os recursos disponíveis de maneira racional para atender mais e melhor as pessoas. No entanto, observa-se pelos dados apresentados no gráfico acima, que os usuários do sistema de saúde atribuem diferentes níveis de importância aos serviços assistenciais. 


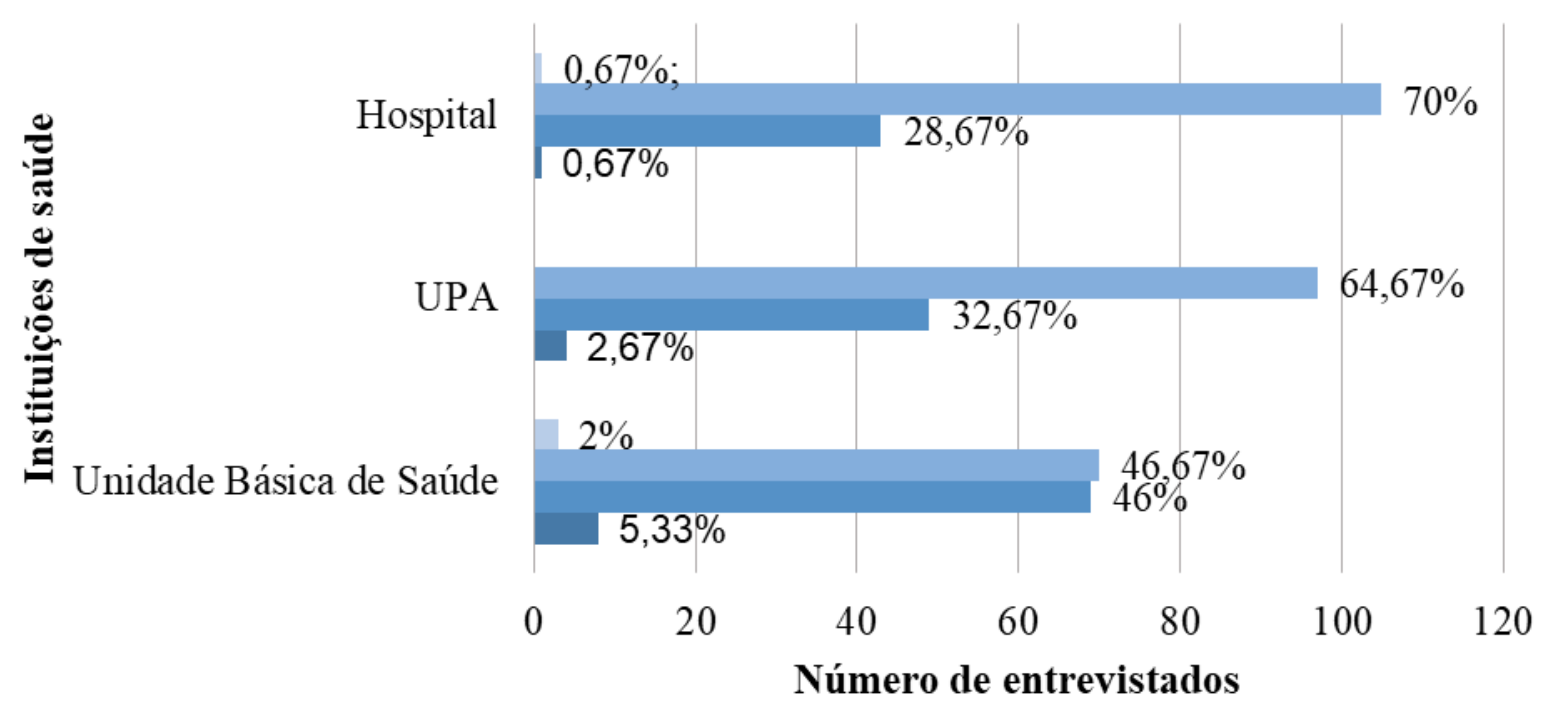

$\square$ Nenhum $\square$ Muito Importante $\quad$ Importante $\quad$ Pouco Importante

Gráfico 2 - Distribuição do grau de importância atribuído às instituições de saúde. Bragança Paulista, 2017. (N=150) (Fonte: Próprio autor).

Pelos dados apresentados no gráfico 3, observa-se que, a maior parte dos entrevistados $(86,67 \%)$ declarou não possuir conhecimento sobre o que é o SUS e uma parcela substancialmente baixa $(13,33 \%)$ indicou a resposta número 3 , sendo esta, a correta ao definir este sistema de saúde. É possível evidenciar que uma parcela importante dos entrevistados (20\%) relaciona o SUS como um sistema direcionado a pessoas que não possuem acesso a hospitais ao escolherem a resposta número 4.

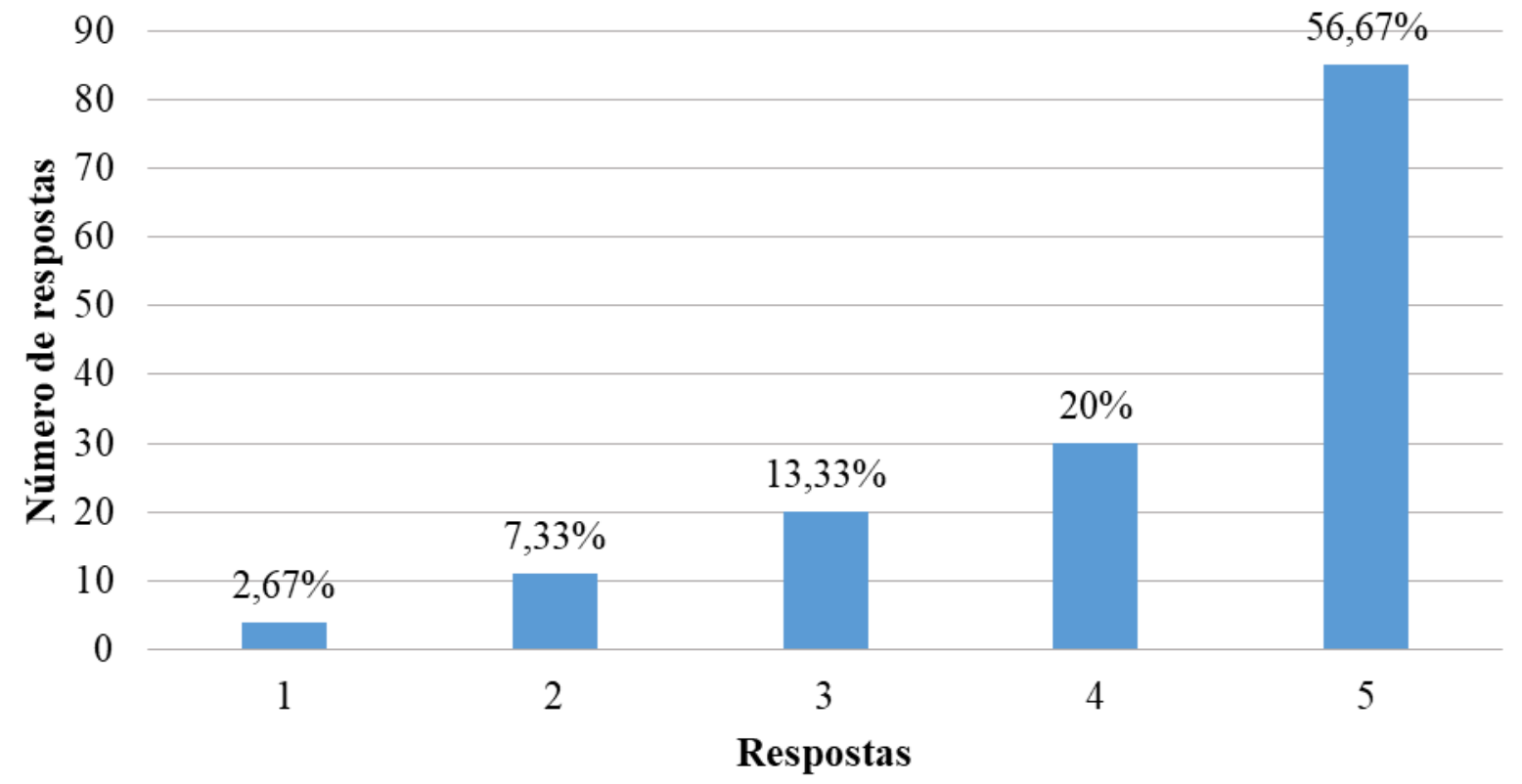

Gráfico 3 - Distribuição das respostas de acordo com o entendimento dos entrevistados sobre o que é o SUS. Bragança Paulista, 2017. ( $\mathrm{N=150).} 1$ - É um sistema de saúde destinado apenas para pessoas que não tem condições financeiras para pagar plano de saúde; 2 - É um sistema único de saúde destinado para todas as pessoas, mas só utiliza quem não tem plano de saúde; 3 -É um sistema de saúde destinado a atender todas as pessoas de maneira gratuita através de ações e serviços que possibilitam a promoção, prevenção, 
tratamento e reabilitação à saúde; 4 - É um sistema de saúde disponibilizado pelo Estado e dirigido a pessoas que não tem acesso a hospitais; 5 - Eu não sei o que é o Sistema Único de Saúde - SUS (Fonte: Próprio autor).

Pereira (2017) questiona o quão é desconhecido o Sistema Único de Saúde - SUS desde sua implantação no país e aponta a necessidade de intervir para que sua identificação como um sistema de saúde se consolide. O mesmo autor em sua pesquisa, demonstrou que dois terços dos entrevistados consideraram não estar bem informados quanto ao SUS, do mesmo modo que, Kawashima et al. (2017) afirmou que o conhecimento da população sobre os princípios relacionados ao SUS demonstra-se insuficiente. Ambas as asserções vão encontro dos resultados da presente pesquisa.

De acordo com Paim (2015), a população nem sempre possui uma ideia correta e clara sobre o SUS e quando questionada sobre o que é o sistema existiriam várias respostas, alguns relacionariam o SUS às filas de postos de saúde e hospitais, outros mencionariam as pessoas em macas nos corredores de serviços de urgência, e muitos alegariam ser o SUS, o atendimento para a camada social pobre, até mesmo, caracterizando-o como um plano de saúde dos pobres.

Importante mencionar a pesquisa do Conselho Nacional de Secretários de Saúde de 2003, que não procurou identificar, como neste estudo, o nível de conhecimento da amostra sobre o que é o Sistema Único Saúde, mas apenas o que a sigla SUS significava, onde os resultados revelaram que apenas 35\% dos entrevistados sabiam indicar com exatidão, o que significa "SUS" e mais de 55\% admitiram não conhecer o significado da sigla.

Observa-se no gráfico 4 que, a maioria dos entrevistados fez uma avaliação positiva dos serviços do SUS ao considerarem como bons $(52,67 \%)$ e excelentes $(11,33 \%)$, no entanto uma pequena minoria avaliou como péssimos $(6,00 \%)$.

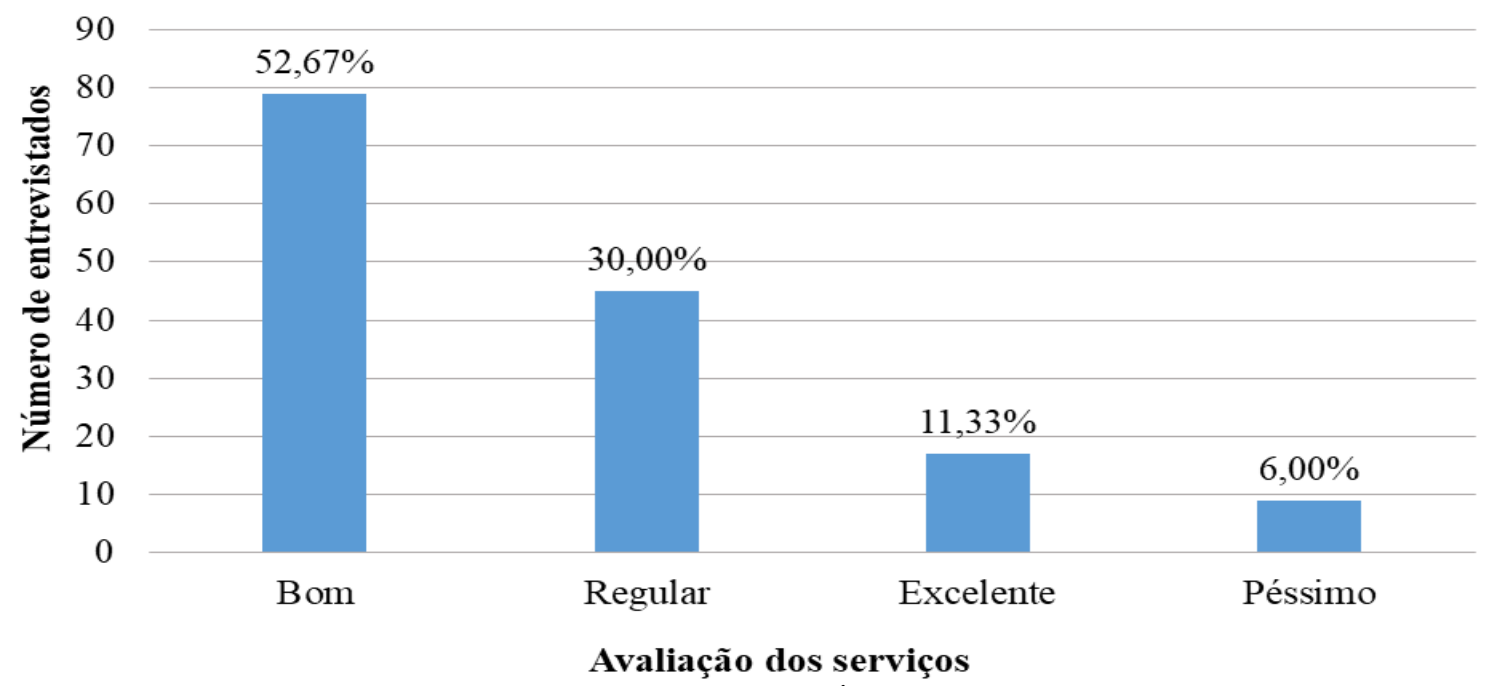

Gráfico 4 - Avaliação dos serviços do Sistema Único de Saúde. Bragança Paulista, 2017. $(\mathrm{N}=150)$ (Fonte: Próprio autor).

Segundo Paim (2015) a Pesquisa de Satisfação dos Usuários do SUS, realizada entre 2011 e 2012, revelou que entre os entrevistados que utilizaram o SUS, 36\% o avaliaram como bom ou muito bom, ao passo que, $29 \%$ o consideraram ruim ou muito ruim. A pesquisa realizada pelo Datafolha em 2014 evidenciou que $19 \%$ dos entrevistados atribuíram nota zero ao SUS, significando péssimo, e $4 \%$ nota dez, denotando ser excelente. Já a pesquisa encomendada pelo Conselho Nacional de Secretários de Saúde - CONASS, no ano de 2003, demonstrou que, o grupo de usuários exclusivos do SUS, $45,2 \%$ considerou que o SUS 
funciona bem/muito bem, já para o grupo de usuários não exclusivos do SUS, esta porcentagem equivaleu a $41,6 \%$.

De maneira geral, observa-se que o SUS é avaliado positivamente pelos usuários, como observado na atual pesquisa.

Conforme os dados apresentados no gráfico 5, 84,67\% dos entrevistados recomendariam os serviços do SUS e $15,33 \%$ não recomendariam.
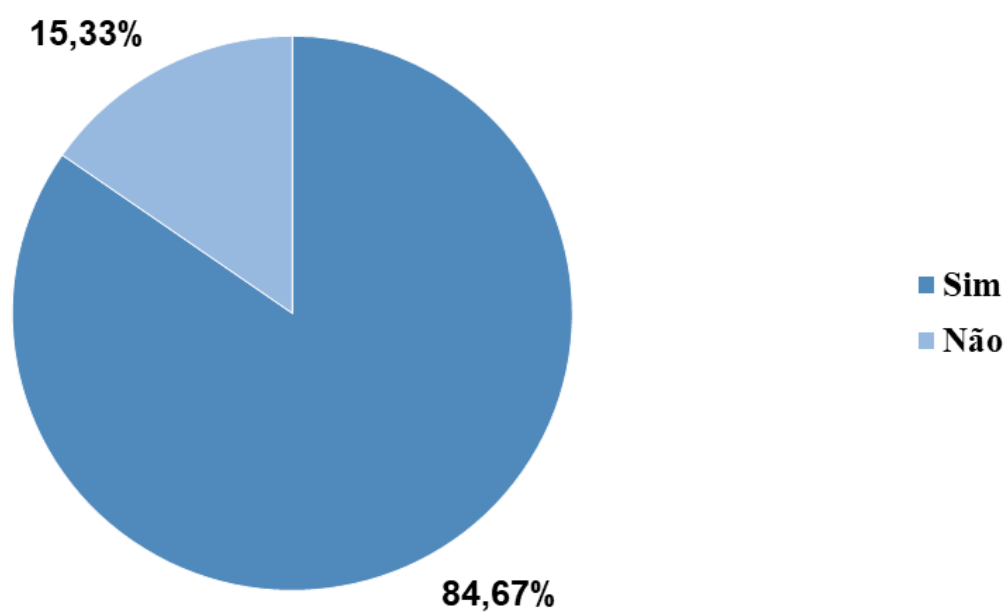

ఐ Não

Gráfico 5 - Percentual de recomendação dos serviços do Sistema Único de Saúde. Bragança Paulista, 2017. ( $\mathrm{N}=150)$ (Fonte: Próprio autor).

Na tabela 2, observa-se que os motivos de maior prevalência para recomendação dos serviços do SUS pelos usuários, foram, o bom atendimento $(36,22 \%)$, a resolutividade $(33,07 \%)$ e existência de pessoas que não possuem condições financeiras para arcar com os cursos de planos de saúde, encontrando-se então, sem opção $(21,26 \%)$. Já os motivos de menor predominância relacionaram, o atendimento ser rápido $(3,15 \%)$, profissionais atuantes serem competentes $(2,36 \%)$, o acesso aos serviços ser um direito da população $(2,36 \%)$ e por existir a necessidade, em algum momento, de procurar atendimento para resolução de problemas de saúde $(1,57 \%)$.

Tabela 2 - Relação dos motivos de recomendação dos serviços do Sistema Único de Saúde. Bragança Paulista, 2017. ( $\mathrm{N}=127)$.

\begin{tabular}{lcc}
\hline Domínio & Frequência & \% \\
\hline Bom atendimento & 46 & 36,22 \\
Resolutividade & 42 & 33,07 \\
Sem recursos financeiros/Sem opção & 27 & 21,26 \\
Atendimento rápido & 04 & 3,15 \\
Direito de atendimento & 03 & 2,36 \\
$\begin{array}{l}\text { Profissionais competentes } \\
\text { Necessidade de atendimento } \\
\text { resolução de problemas de saúde }\end{array}$ & 03 & 2,36 \\
Total para & 02 & 1,57 \\
\hline
\end{tabular}

Fonte: Próprio autor.

De acordo com os resultados apresentados na tabela 3, as maiores razões de não indicação dos serviços do SUS pelos usuários relacionaram, o atendimento ruim $(43,48 \%)$ e demorado $(21,74 \%)$, bem como, os serviços não serem resolutivos $(17,39 \%)$. A razão do serviço privado ser melhor $(4,35 \%)$, foi o menor motivo pela não recomendação do SUS. 
Tabela 3 - Relação dos motivos para não recomendação dos serviços do Sistema Único de Saúde. Bragança Paulista, 2017. (N=23).

\begin{tabular}{lcc}
\hline Domínio & Frequência & \% \\
\hline Atendimento ruim & 10 & 43,48 \\
Atendimento demorado & 05 & 21,74 \\
Sem resolutividade & 04 & 17,39 \\
Profissionais despreparados e desmotivados & 03 & 13,04 \\
Serviço privado é melhor & 01 & 4,35 \\
\hline Total & 23 & $100 \%$ \\
\hline
\end{tabular}

Fonte: Próprio autor.

Fadel e Regis Filho (2009) afirmam que a percepção da qualidade dos serviços públicos pelos usuários se baseia em critérios objetivos e subjetivos, e representa uma iniciativa para o desenvolvimento de ações que possam conduzir a melhorias. Berwick (1994 apud SANTOS; LACERDA, 1999, p. 44) complementa que a qualidade da assistência nos serviços de saúde pode ser estabelecida como satisfação das necessidades dos clientes. Conforme Silva e Formigli (1994), a satisfação é compreendida como a percepção e julgamento que o usuário possui sobre os serviços de saúde, e por sua vez, constitui um elemento essencial para a avaliação da qualidade de tais serviços. Paim (2015) ao considerar o inquérito epidemiológico no Brasil em 2003, a avaliação global da assistência de saúde no país indicou $58 \%$ dos entrevistados estavam insatisfeitos e $27 \%$ satisfeitos.

$\mathrm{O}$ presente estudo demonstrou que a maior parte dos entrevistados recomendaria os serviços do Sistema Único de Saúde, conforme gráfico 5, e dentre as razões, o bom atendimento, ser resolutivo e haver pessoas que não possuem recursos financeiros para obter um plano de saúde, encontrando-se, então, sem opção para atendimento, foram as mais prevalentes. A resolutividade foi um dos motivos de satisfação pelos usuários no estudo de Moimaz et al. (2010), com um percentual de 72, constatando também, a confiança nos profissionais de saúde da rede pública, num total de 69,5\%. Importante mencionar, neste contexto, a fala de Paim (2015), ao afirmar que, quase $98 \%$ da população que necessitou de serviços de saúde, foram atendidos pelo SUS.

A minoria dos entrevistados referiu não recomendar os serviços do SUS, de acordo com o gráfico 5, e um dos motivos já relatados foi devido os serviços não serem resolutivos. A pesquisa de Moimaz et al. (2010) constatou como razões para não resolubilidade do sistema, a ausência de medicamentos, vagas insuficientes e demora para atendimento, complicações no sistema de referência e contra referência e poucos profissionais em atividade. O mesmo autor ainda observou que 57,6\% dos entrevistados referiram existir fila para o atendimento. O tempo de espera foi um fator, também mencionado na pesquisa do Conselho Nacional de Secretários de Saúde - CONASS, de 2003, para a insatisfação dos usuários do sistema ao aludirem a demora tanto para o atendimento, filas de espera, para marcar consultas e recepção.

Para Carvalho (1996 apud SANTOS; LACERDA, 1999, p. 44) o desempenho do Sistema Único de Saúde encontra-se distante do modelo proposto, no qual a população deve ter suas necessidades rapidamente atendidas. E ainda complementa, que problemas como, ausência de recursos financeiros, desqualificação dos recursos humanos e existência de programas de saúde excluídos de uma política social, repercutem no baixo índice de satisfação dos usuários do sistema.

O presente estudo permitiu identificar as principais razões que levam os usuários do SUS a utilizarem os serviços de urgência e emergência como porta de entrada preferencial ao sistema para a resolução de agravos de saúde não urgentes. Os motivos de maior relevância 
relacionaram o acreditar que o serviço de urgência e emergência, a exemplo a Unidade de Pronto Atendimento, possui maior resolutividade para os problemas de saúde, o caráter de atendimento apenas agendado pelas UBS/ESF que compõem a rede básica de saúde, bem como, o horário de funcionamento limitado das mesmas. A análise dos resultados em questão, possibilitou a compreensão de que a Atenção Básica não encontra-se ordenada para o atendimento da demanda espontânea. $\mathrm{O}$ agendamento não assegura a assistência à saúde em tempo minimamente ideal, quando se trata de problemas que comprometam a saúde, bem como, a qualidade de vida de uma determinada pessoa, visto que as agendas disponíveis apresentam um tempo de espera longo na percepção dos usuários. Tal situação é acentuada pelo horário limitado de atendimento pelas UBS/ESF, não abrangendo, a exemplo, aquele usuário trabalhador com jornada de trabalho de 40 horas semanais e que ao final do expediente depara-se com as portas da $\mathrm{AB}$, ditas entrada preferencial, fechadas.

As diretrizes e propósitos do SUS, apesar de mostrarem-se admiráveis no papel desde sua criação, apresentam incoerências no desenvolvimento, o que por sua vez, podem estar relacionadas à insuficiência de recursos humanos e econômicos, despreparo dos profissionais de saúde em atuarem em um serviço público, vulnerabilidades de gestão, uso inapropriado dos serviços pela sociedade ou mesmo a incompreensão pelos usuários e profissionais de saúde, tanto gestores como assistenciais, sobre os desígnios do SUS e proposta de trabalho.

A maior parte da população do presente estudo referiu saber que a $A B$ é capaz de resolver a maioria dos problemas de saúde e ser a porta de entrada preferencial no sistema de saúde. Ainda que exista tal conhecimento, os usuários têm a tendência de dirigirem-se a unidades de urgência e emergência para a resolução de agravos à saúde não urgentes, frente as limitações que a $A B$ apresenta. Sendo assim, a $A B$ assume um grau de importância e de resolutividade consideravelmente inferiores aos demais serviços assistenciais e ao que é esperado. É perceptível que o usuário irá atribuir maior ou menor relevância à um serviço de saúde quando este consegue satisfazer de maneira eficiente suas necessidades atuais de saúde e diante daquilo que o mesmo julga ser um bom atendimento. A crença existente entre a população de que a assistência pautada na medicalização e exames imediatos, melhor atende as necessidades de saúde, minimiza as potencialidades da $\mathrm{AB}$, bem como, seu uso prioritário.

As evidências da pesquisa orientam a reflexão sobre quais são os desafios atuais da Atenção Básica frente às suas limitações e fragilidades, para o fortalecimento do acesso e resolutividade da assistência ofertada, de modo que a população a utilize como porta preferencial, capaz de atender suas necessidades de saúde, e por fim, exerça verdadeiramente seu papel de forma ativa e coerente com a proposta do modelo assistencial. Ademais, a pesquisa permite ponderar a necessidade do poder público e de profissionais em reafirmar o papel do SUS proporcionando condições para que este sistema de saúde atue em equilíbrio com seus princípios e diretrizes, recursos disponíveis e contemplando as necessidades que a população possa apresentar no campo da saúde

A população usufrui de um dos maiores sistemas públicos de saúde existentes sem o conhecimento amplo sobre o que ele realmente seja. O acesso desta população ao sistema de saúde, de forma que, suas necessidades sejam atendidas da melhor maneira possível, exige o saber sobre as potencialidades dos serviços disponibilizados. O conhecimento permite a definitiva concretização de direitos constitucionais, a possibilidade de resolução de problemas e identificação das necessidades no processo saúde/doença, assim como, extingue falsos paradigmas sobre o sistema de saúde instalados nas sociedades com o passar do tempo.

Apesar dos entraves e possíveis contestações, a maioria dos usuários valoriza o sistema que tem à sua disposição como política pública de saúde, considerando os resultados pertinentes ao nível de satisfação e recomendação do sistema, contudo, dentre as falas de recomendação do sistema de saúde, a necessidade de melhorias foi aludida. 


\section{CONCLUSÃO}

O presente estudo alcançou os objetivos propostos e teve sua importância, uma vez que, permitiu discutir uma realidade do sistema de saúde, a inversão da porta de entrada preferencial ao mesmo, que por sua vez, contrapõe um de seus preceitos organizativos e acarreta inconsistências assistenciais no processo saúde/doença. Sendo assim, acredita-se que o presente estudo poderá contribuir na avaliação do desempenho atual do sistema de saúde, dos profissionais de saúde e gestores, mobilizando iniciativas que levam ao remodelamento da atenção à saúde, com base nos princípios e diretrizes outorgados constitucionalmente, possibilitando o fortalecimento da Atenção Básica, o desenvolvimento harmonioso e adequado do sistema de saúde maximizando a resolutividade dos problemas e necessidades apresentados pelo indivíduo e comunidade, aliado a um melhor conhecimento da população sobre o que o Sistema Único de Saúde representa, considerando suas conquistas e potencialidades.

\section{REFERÊNCIAS}

BARISTELA, S.; GUERREIRO, N. P.; ROSSETTO, E. G. Os motivos de procura pelo Pronto Socorro Pediátrico de um Hospital Universitário referidos pelos pais ou responsáveis. Ciências Biológicas e da Saúde, Londrina, 29 v., 2 n., p. 121 - 130, jul/dez. 2008.

Disponível em:

<http://www.uel.br/proppg/portal/pages/arquivos/pesquisa/semina/pdf/semina_29_2_20_28.p df $>$. Acesso em: 06 out. 2017.

\section{BERWICK, D. M. Melhorando a qualidade dos serviços médicos, hospitalares e da} saúde. São Paulo: Markon Books, 1994.

BRASIL. Casa Civil. Decreto $\mathbf{N}^{\mathbf{0}} \mathbf{7 . 5 0 8}$, de 28 de Junho de 2011. Regulamenta a Lei no 8.080, de 19 de setembro de 1990, para dispor sobre a organização do Sistema Único de Saúde - SUS, o planejamento da saúde, a assistência à saúde e a articulação interfederativa, e dá outras providências. Disponível em: <http://www.planalto.gov.br/ccivil_03/_ato20112014/2011/decreto/d7508.htm>. Acesso em: 27 abr. 2017.

. Conselho Nacional de Secretários de Saúde. A saúde na opinião dos brasileiros. Conselho Nacional de Secretários de Saúde. Brasília: CONASS, 2003. Disponível em: <http://bvsms.saude.gov.br/bvs/publicacoes/opiniao_brasileiros1.pdf>. Acesso em: 28 set. 2017.

Ministério da Saúde. O SUS de A a Z. Ministério da Saúde. Conselho Nacional de Secretarias Municipais de Saúde. 3 ed. Brasília: Ministério da Saúde, 2009. Disponível em: <http://bvsms.saude.gov.br/bvs/publicacoes/sus_az_garantindo_saude_municipios_3ed_p1.pd f>. Acesso em: 06 abr. 2017.

CAMERRO, A.; ALVES, E. C.; CAMERRO, N. M. M. da S.; NOGUEIRA, L. D. P. Perfil do atendimento de serviços de urgência e emergência. Revista Fafibe On-Line, Bebedouro São Paulo, p. 515 - 524, 2015. Disponível em:

$<$ http://unifafibe.com.br/revistasonline/arquivos/revistafafibeonline/sumario/36/10112015195 658.pdf>. Acesso em: 31 mar. 2017.

CARVALHO, R. 10 anos de SUS: aniversário sem comemoração. Revista Prodoctor Resident, São Paulo, 3 v., 5 n., p. 8-9, set/lout. 1996. 
CHAGAS, H. M. de A.; VASCONCELLOS, M. da P. C. Quando a porta de entrada não resolve: análise das unidades de saúde da família no município de Rio Branco, Acre. Saúde soc., São Paulo, 22 v., 2 n., p. 377-388, jun. 2013. Disponível em: <http://dx.doi.org/10.1590/S0104-12902013000200010>. Acesso em: 27 set. 2017.

DATAFOLHA. Opinião dos brasileiros sobre o atendimento na área de saúde. 2014. Disponível em: <http://portal.cfm.org.br/images/PDF/apresentao-integra-datafolha203.pdf>. Acesso em: 06 out. 2017.

FADEL, M. A. V.; REGIS FILHO, G. I. Percepção da qualidade em serviços públicos de saúde: um estudo de caso. Rev. Adm. Pública, Rio de Janeiro, 43 v., 1 n., p. 7-22, fev. 2009. Disponível em: <http://dx.doi.org/10.1590/S0034-76122009000100002>. Acesso em: 06 out. 2017.

FREIRE, A. B.; FERNANDES, D. da L.; MORO, J. da S.; KNEIPP, M. M.; CARDOSO, C. M.; LIMA, S. B. S. de. Serviços de urgência e emergência: quais os motivos que levam o usuário aos pronto-atendimentos? Santa Maria: Saúde, jan/jul. 2015. 41 v., 1 n., p. 195 200. Disponível em:

<https://periodicos.ufsm.br/index.php/revistasaude/article/viewFile/15061/pdf >. Acesso em: 31 mar. 2017.

GOMIDE, M. F. S.; PINTO, I. C.; FIGUEIREDO, L. A. de. Acessibilidade e demanda em uma Unidade de Pronto Atendimento: perspectiva do usuário. Acta paul. enferm., São Paulo, 25 v., 2 n., p. 19 - 25, 2012. Disponível em: <http://dx.doi.org/10.1590/S0103$21002012000900004>$. Acesso em: 31 mar. 2017.

KAWASHIMA, M. C. dos S.; FERREIRA, M. V.; SILVA, P. C. da; BOAVENTURA, A. P.; ALMEIDA, J. B. de. O Desconhecido SUS: O Conhecimento da População de uma Cidade do Vale do Paraíba. Universidade do Vale do Paraíba. Disponível em: <http://www.inicepg.univap.br/cd/INIC_2007/trabalhos/saude/inic/INICG00420_01O.pdf>. Acesso em: 09 out. 2016.

MARQUES, G. Q.; LIMA, M. A. D. da S. Demandas de usuários a um serviço de pronto atendimento e seu acolhimento ao sistema de saúde. Rev. Latino-Am. Enfermagem, Ribeirão Preto, 15 v., 1 n., p. 13-19, fev. 2007. Disponível em: <http://dx.doi.org/10.1590/S0104-11692007000100003>. Acesso em: 29 set. 2017.

MOIMAZ, S. A. S.; MARQUES, J. A. M.; SALIBA, O.; GARBIN, C. A. S.; ZINA, L. G.; SALIBA, N. A. Satisfação e percepção do usuário do SUS sobre o serviço público de saúde. Physis, Rio de Janeiro, 20 v., 4 n., p. 1419-1440, dez. 2010. Disponível em: <http://dx.doi.org/10.1590/S0103-73312010000400019>. Acesso em: 06 out. 2017.

MONTEIRO, A. C. de P.; SILVA, A. de A. A.; CABRAL, M. C. Estudo de demanda de um serviço de pronto atendimento no município de Recife - PE. 2008. 52 f. Monografia Gestão de Sistemas e Serviços de Saúde, Centro de Pesquisa Aggeu Magalhães, Recife, 2008. Disponível em: <http://www.cpqam.fiocruz.br/bibpdf/2008monteiro-acp.pdf>. Acesso em: 06 out. 2017.

NARVAI, P. C. Integralidade na atenção básica à saúde. Integralidade? Atenção? Básica? Rio de Janeiro: ABORJ/ANS/UNESCO, 2005. p. 28 - 42. Disponível em: 
<http://www.idisa.org.br/img/File/IntegralidadeAtencaoBasica2005.pdf>. Acesso em: 01 abr. 2017.

OLIVEIRA, M. L. F. de; SCOCHI, M. J. Determinantes da utilização dos serviços de urgência/emergência em Maringá (PR). Revista Ciência, Cuidado e Saúde, Maringá, 1 v., 1 n., p. 123-128, 2002. Disponível em:

<http://www.periodicos.uem.br/ojs/index.php/CiencCuidSaude/article/view/5666/3605>. Acesso em: 29 set. 2017.

PAIM, J. S. O que é o SUS. Rio de Janeiro: Fiocruz, 2015. E-book. Disponível em: <http://www.livrosinterativoseditora.fiocruz.br/sus/>. Acesso em: 21 abr. 2017.

PEREIRA, J. de J. T. Avaliação do nível de conhecimento sobre o SUS numa amostra populacional usuária. Secretaria de Estado da Saúde de Minas Gerais. Disponível em: <http://encipecom.metodista.br/mediawiki/images/9/9c/GT4_-_Avaliacao_do_nivelJoao.pdf>. Acesso em: 09 out. 2016.

PIRES, M. R. G. M.; GÖTTEMS, L. B. D.; CUPERTINO, T. V.; LEITE, L. S.; VALE, L. R. do; CASTRO, M. A. de; LAGE, A. C. A.; MAURO, T. G. da S. A utilização dos serviços de atenção básica e de urgência no SUS de Belo Horizonte: problema de saúde, procedimentos e escolha dos serviços. Saúde soc., São Paulo, 22 v., 1 n., p. 211-222, mar. 2013. Disponível em: 〈http://www.scielo.br/pdf/sausoc/v22n1/19.pdf>. Acesso em: 20 set. 2017.

ROCHA, A. F. S. Determinantes da procura de atendimento de urgência pelos usuários nas unidades de pronto atendimento da secretaria municipal de saúde de Belo Horizonte. 2005. 98 f. Dissertação de Mestrado - Enfermagem, Universidade Federal de Minas Gerais, Belo Horizonte, 2005. Disponível em: <http://www.enf.ufmg.br/pos/defesas/310M.PDF>. Acesso em: 06 out. 2017.

SANTOS, S. R. dos; LACERDA, M. do C. N. de. Fatores de satisfação e insatisfação entre os pacientes assistidos pelo SUS. Rev. bras. enferm., Brasília, 52 v., 1 n., p. 43-53, mar. 1999. Disponível em: <http://dx.doi.org/10.1590/S0034-71671999000100006>. Acesso em: 06 out. 2017.

SILVA, L. M. V. da; FORMIGLI, V. L. A. Avaliação em saúde: limites e perspectivas. Cad. Saúde Pública, Rio de Janeiro, 10 v., 1 n., p. 80-91, mar. 1994. Disponível em: <http://dx.doi.org/10.1590/S0102-311X1994000100009>. Acesso em 01 nov. 2017.

SOUZA, C. C. de; ARAÚJO, F. A.; CHIANCA, T. C. M. Produção científica sobre a validade e confiabilidade do Protocolo de Manchester: revisão integrativa da literatura. Rev. esc. enferm. USP, São Paulo, 49 v., 1 n., p. 144 - 151, fev. 2015. Disponível em: <http://dx.doi.org/10.1590/S0080-623420150000100019>. Acesso em: 01 abr. 2017. 